\title{
EDITORIAL
}

\section{Introduction: systemic effects in chronic obstructive pulmonary disease}

\author{
E.F.M. Wouters
}

\begin{abstract}
According to the definition of the European Respiratory Society, chronic obstructive pulmonary disease (COPD) is a disorder characterised by reduced maximum expiratory flow and slow forced emptying of the lungs [1]. The clinical syndrome of COPD, manifested clinically by dyspnoea, cough and impaired exercise tolerance, encompasses different disease conditions, varying from chronic obstructive bronchitis with obstruction of small airways, to emphysema characterised by enlargement of air spaces and destruction of lung parenchyma, loss of lung elasticity, and closure of small airways. The outcome of this differentiation in COPD subtypes in the natural history of the COPD process still remains largely unexplored although imaging strategies easily allow a better characterisation of the pathological consequences of this disorder. Most attention is still focused on adequate diagnosis of COPD per se by lung function testing especially in the primary care setting.
\end{abstract}

The association of an abnormal inflammatory response of the lungs to noxious particles or gases with airflow limitation in COPD indicates the critical role of the inflammatory process in the pathogenesis of COPD. However, despite the important role of inflammation in the pathophysiology of COPD, the inflammatory process itself is still less well defined, no data of the inflammatory process during the natural course of the disease are yet available and no information is available about differentiation of inflammation in the different COPD phenotypes. Furthermore, the role of systemic inflammation as well as the contributing mechanisms is largely overlooked in the assessment of the COPD patients. Lacking this critical information, it can be questioned if it will be possible to modulate progression of the disease process, which has been considered as the most important treatment goal in COPD for many decades.

International guidelines have stressed the important role of an integrated management of the COPD patient in order to improve exercise tolerance and health status, to relieve symptoms and to prevent complications and exacerbations. The extent to which these goals can be realised by treatment of the local organ impairment is still limited [2].

Other largely nonpharmacological intervention strategies such as pulmonary rehabilitation demonstrated that symptomatic COPD patients benefit considerably from such comprehensive treatment programmes. Indeed, half a century ago, body composition and especially body weight was already retained as an important discriminator to describe different COPD classifications and a lot of studies demonstrated the important role of body weight maintenance in the outcome of these patients $[3,4]$. New indirect techniques to assess body composition indicate that weight loss in COPD can be the

Correspondence: University Hospital Maastricht, Dept of Pulmonology, P.O. Box 5800, 6202 AZ Maastricht, The Netherlands. Fax: 31 433877087. E-mail: EWO@slon.azm.nl result of a loss in fat mass, a loss in fat-free mass (FFM) or both [5]. FFM is generally considered as a good indirect estimate of body cell mass, the actively metabolising and contracting tissue. During the last decade, muscle wasting and skeletal muscle dysfunction were identified as a major determinant in the morbidity and mortality experienced by COPD patients, stressing the important role of these secondary effects of COPD. This Supplement of the European Respiratory Journal extensively reviews the present knowledge of respiratory and skeletal muscle function and metabolism in COPD as well as processes involved such as systemic inflammation and disturbances in oxidant-antioxidant metabolism as well as endocrine disturbances. The complexity of fluid homeostasis in COPD patients suffering from cor pulmonale further illustrates the important role of endocrine mechanisms in the pathophysiology of COPD. Other systemic effects of COPD such as vascular changes and osteoskeletal effects are extensively reviewed in this supplement.

Adequate assessment of chronic obstructive pulmonary disease patients have to include consideration of these systemic components of the disease. Optimal therapeutic targeting depends on a clear understanding of the precise mechanisms of these complex processes. At present, treatment of these systemic effects is important to reach widely accepted management goals. New therapeutic strategies targeting these systemic effects may offer new perspectives in the management of chronic obstructive pulmonary disease patients in order to achieve a better health status and prognosis for the majority of these very disabled patients. The present possibilities of nutritional and metabolic modulation are therefore also reviewed in this supplement.

\section{References}

1. Siafakas NM, Bouros N. Management of acute exacerbation of chronic obstructive pulmonary disease. Eur Respir Mon 1998; 3(7): 264-277.

2. Curtis JR, Deyo RA, Hudson LD. Pulmonary rehabilitation in chronic respiratory insufficiency. 7. Health-related quality of life among patients with chronic obstructive pulmonary disease. Thorax 1994; 49: 162-170.

3. Landbo C, Prescott E, Lange P, Vestbo J, Almdal TP. Prognostic value of nutritional status in chronic obstructive pulmonary disease. Am J Respir Crit Care Med 1999; 160: $1856-1861$.

4. Schols AM, Slangen J, Volovics L, Wouters EF. Weight loss is a reversible factor in the prognosis of chronic obstructive pulmonary disease. Am J Respir Crit Care Med 1998; 157: 1791-1797.

5. Schols AM, Soeters PB, Dingemans AM, Mostert R, Frantzen PJ, Wouters EF. Prevalence and characteristics of nutritional depletion in patients with stable COPD eligible for pulmonary rehabilitation. Am Rev Respir Dis 1993; 147: $1151-1156$. 